\title{
BENEFICIOS DEL MODELO AS A SERVICE EN LAS PYMES
}

\section{Julio Rodríguez Arquiñigo}

\begin{abstract}
Resumen
Uno de los aspectos que actualmente causa mayor preocupación en las pymes son los gastos de gestión en los que incurren durante los primeros años de actividad, mientras buscan su estabilidad y posterior crecimiento. Cloud computing (computación en la nube) se han convertido en las palabras de moda en los últimos años, en especial el modelo As a service (Como servicio), ya que mediante dicho modelo las pymes pueden acceder, en forma más económica, a servicios que tradicionalmente han sido costosos, debido a la cantidad y requerimientos de los equipos involucrados. Este modelo brinda la facilidad de disponer de un servicio "transparente", que involucre equipos de cómputo multimarca, en formato de renta y con escenarios de renovación tecnológica, con lo cual se disminuye la obsolescencia de infraestructura, ya que se prescinde de equipos propios, y a su vez se logra una reducción de los activos asociados. Debido al potencial impacto que tiene el cloud computing en numerosas aplicaciones de negocios, así como en nuestra vida cotidiana, se puede afirmar, sin duda, que la computación en la nube llegó para quedarse. Por lo tanto, es necesario revisar los beneficios de consolidar servicios y acceder a dicho modelo.
\end{abstract}

Palabras clave: cloud computing, gestión de pymes, as a service

\section{Benefits of the model "as a service" in the SMB}

\section{Summary}

One of the aspects that causes the most concern in the SMBs are the costs of managing incurred during the first years of activity, while searching for its stability and subsequent growth. Cloud computing, has become a commonly used word in the past few years, in particular the model "As a service", because it is through this model that SMBS can access, in a more economic way to services that have traditionally been expensive. This model provides the ease of having a service that involves multi-brand computers, in the form of income and with scenarios of technological renewal; lessening the obsolescence of infrastructure, since it prescinds from own equipment. Due to the impact of cloud computing in the business applications, and in our daily lives, it can be said, that this model has come to stay; therefore, it is necessary to carry out a review of the benefits for companies.

Key words: cloud computing, management of SMBs, as a service 


\section{Introducción}

Nuestro país es una plaza para los negocios que ha ido mejorando con el paso del tiempo, y esto sin duda favorece la aparición de emprendimientos, por lo cual se debe tener en cuenta que una pyme es vulnerable a muchas variables de nuestro entorno de mercado, pero además existen factores que están dentro de casa, como los temores propios del emprendedor que inicia su proyecto, quien se desencanta al ver de qué manera lo que parecía un gran sueño, se desvanece con el pasar del tiempo.

Por lo tanto, superar el primer año de actividades es uno de los grandes retos para toda pequeña empresa que busca insertarse y afianzarse en el mercado, pero este es solo uno de ellos. Sobrevivir y destacar dependerá de haber desarrollado un plan de negocios estructurado, del modelo de gestión, del orden y el compromiso con el trabajo, de reconocer en su cadena de valor aquella ventaja competitiva, la cual deberá ser sostenible en el largo plazo, ajustando la relación precio-costo-valor para garantizar el éxito, pero por sobre todo no renunciar al sueño inicial. Algunas cifras de constitución de las pymes, obtenidas por el Instituto Nacional de Estadística e Informática (INEI), se presentan en el anexo, al final del artículo.

Uno de los principales problemas de las pymes es quizás la carencia de la información adecuada y oportuna para su gestión, o tal vez una lectura o interpretación a destiempo de la información disponible. En la búsqueda de querer subsistir se centran en la operación pero no la monitorean; sin embargo, surge la siguiente reflexión: ¿por qué las pymes se sienten excluidas de acceder a las herramientas tecnológicas? Existen varios factores que responden esta interrogante, como la informalidad, el desconocimiento de las tecnologías existentes y los altos costos que deberían enfrentar para acceder a equipos que les permitan operar como las grandes empresas, por ejemplo una central telefónica física (PBX) o un equipo de seguridad perimetral para su red, entre otros.

Por ello, surge el concepto de servicio gestionado (managed service) y este va avanzando firmemente; y es que "gestionar" significa poder administrar equipamiento de tecnología avanzada en los data centers de las empresas de telecomunicación para poder desarrollar servicios dirigidos para consumidores inexpertos, quienes no requieren conocer dicha tecnología, pero que podrían acceder a sus beneficios. Justamente, las empresas de telecomunicaciones ofrecen dichos servicios como valor agregado a sus enlaces de voz, datos e Internet, con el propósito de poder fidelizar a sus clientes y captar otros nuevos.

Se ha hablado y escrito mucho acerca de la tecnología cloud computing, en especial por expertos en la industria, líderes empresariales e independientes. 
Las soluciones en la nube permiten canalizar aquella solución lejana y acercarlas a las pequeñas empresas; por ejemplo, tener correo con dominio propio por una renta mensual mínima, como el servicio brindado por Google, que tiene un buen nivel de servicio y del cual difícilmente se podría presentar queja. Anteriormente, se debía adquirir un dominio de correo, luego, contratar un proveedor de correo, y finalmente configurar los clientes de los correos, Outlook entre ellos, pues las interfaces web no eran tan intuitivas como ahora. Los tiempos han cambiado; ahora Google lo tiene todo en un solo sitio desde cinco dólares mensuales por usuario.

Esto favorece a la pequeña y mediana empresa, ya que contribuye a mejorar su imagen y le brinda herramientas para competir en un mercado dinámico y competitivo. Asimismo, el solo hecho de que la pyme disponga de un POS (point of sale) le permite realizar operaciones a través de las compañías de medios de pago, ser vista de mejor forma, además de lograr un aumento de sus ingresos.

Los riesgos a los que están expuestas hacen que las pymes busquen asociarse por diversos motivos. Sin embargo, cada vez más estas van internalizando la necesidad de la tecnología en su negocio.

Por lo expuesto, cloud computing no resulta ser solo una moda pasajera, sino que pretende quedarse instaurada en el medio empresarial. Entonces, ¿qué es exactamente cloud computing?; desde la perspectiva del usuario, es una tecnología que proporciona un medio para adquirir servicios informáticos sin necesidad de tener una comprensión profunda de los aspectos tecnológicos involucrados. Desde la perspectiva de una organización, el cloud computing ofrece servicios para las necesidades del consumidor y los negocios de una manera simple, brindando transparencia en la escalabilidad y calidad de servicio, e impulsando la innovación y la adecuada toma de decisiones. De acuerdo a lo publicado por International Data Group (IDG) (2013), se señala que la empresa Gartner INC define a la computación en la nube como un estilo de informática, donde los recursos de tecnología de información son entregados como un servicio, a clientes externos, mediante el uso de tecnologías de Internet.

\section{Modalidades de As a service}

Previo al análisis de las modalidades del modelo As a service, se presenta la infraestructura digital framework utilizada en el cloud computing, la misma que se puede apreciar en la figura 1. 
Figura 1. Framework utilizado en cloud computing

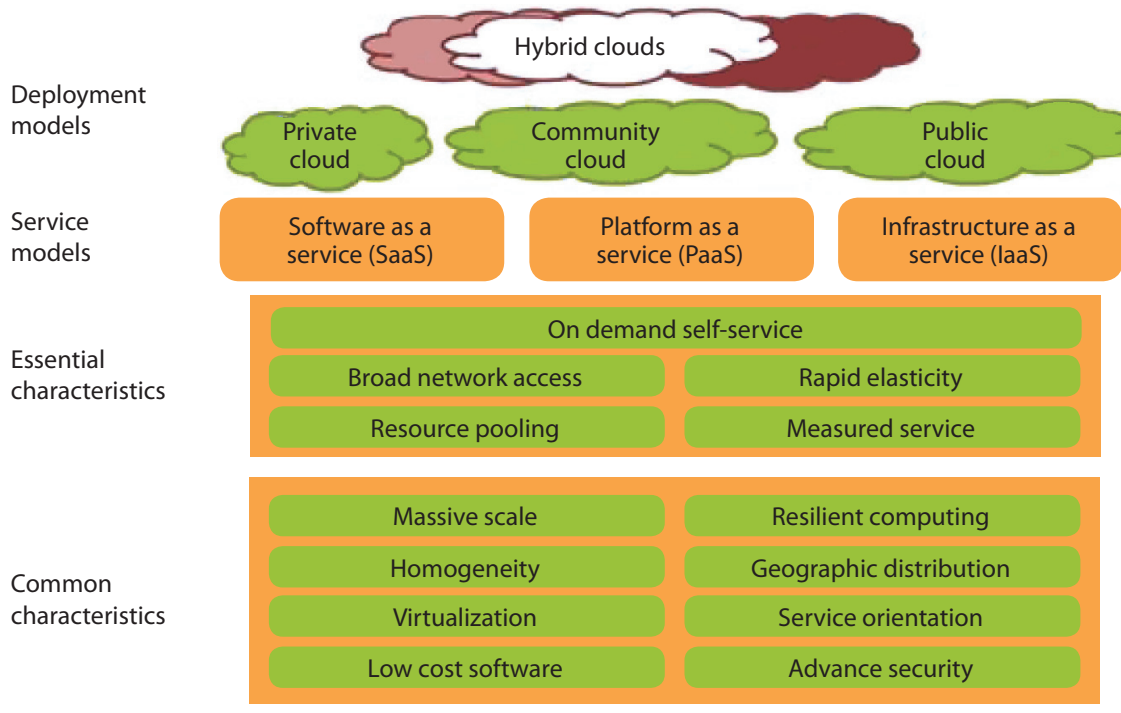

Fuente: Carlo, Biancolino y Maccari, 2013, p. 180.

En la tabla 1 se presenta la lista de las modalidades existentes del modelo As a service, así como los tipos de nubes asociados a cada una de ellas.

Tabla 1. Tipos de modelos As a service y nubes asociadas

\begin{tabular}{|c|c|}
\hline $\begin{array}{c}\text { Modalidades As a service } \\
\text { (laaS) }\end{array}$ & Tipos de nubes \\
\hline $\begin{array}{c}\text { Nnfrastructure as a service } \\
\text { Platform as a service (PaaS) }\end{array}$ & Nube comunitaria \\
\hline Software as a service (SaaS) & Nube privada \\
\hline Everything as a service (XaaS) & Nube híbrida \\
\hline
\end{tabular}

Fuente: Adaptado de Yrigoyen \& Torres, 2011, p. 14.

Como se mencionó anteriormente, con cada uno de estos modelos de servicio cloud ciertas responsabilidades son trasladadas al proveedor del servicio en la nube, permitiendo a los consumidores centrarse más en las necesidades del negocio y menos en las tecnologías subyacentes. Parte importante en el desarrollo de estas soluciones descansa sobre los operadores de telefonía e integradores, pues ellos tienen la infraestructura adecuada con la cual brindan una oferta diferenciada a un costo realmente atractivo para la pequeña empresa. 


\subsection{Infrastructure as a service (laaS)}

Aloja la infraestructura de cómputo necesaria en un lugar remoto, por lo general en data centers, lo cual permite que los clientes ya no cuenten con hardware de estante y no requieran de condiciones para el almacenamiento de este. De igual manera, no se requiere contratar personal para administrar este tipo de equipos, solo bastaría contar con el servicio de Internet. Los operadores de telefonía les facturan a la pyme una renta mensual adicional muy parecida a los consumos de agua o electricidad.

\subsection{Platform as a service (PaaS)}

Representa un nivel superior, con el cual se provee a los usuarios, de manera remota, de un sistema operativo, base de datos, servidor de aplicaciones y lenguaje de programación. De esta manera, los usuarios pueden dedicarse al desarrollo de software sobre la plataforma, sin necesidad de instalar, administrar y actualizar los sistemas operativos, como Windows u otros. PaaS también se encarga del escalamiento, tolerancia a fallos y muchas otras técnicas y consideraciones de diseño, para que los desarrolladores puedan centrarse en las aplicaciones del negocio.

\subsection{Software as a service (SaaS)}

Es el máximo nivel de abstracción. Con SaaS, la aplicación o servicio se entrega en la web a través de un navegador o a través de un programa (API). En este modelo de servicio, el consumidor solo necesita concentrarse en la administración de los usuarios al sistema. SaaS es muy común para aplicaciones de tipo competencia complementarios como gestión de la relación cliente (CRM), planificación de recursos (ERP), aplicaciones de recursos humanos, aplicaciones financieras y contables. Ahora las compañías podrían dejar de entregar software sobre la red interna, optando por un modelo SaaS, donde el software está disponible 24/7 vía Internet. En este modelo, el software es actualizado en un solo lugar y disponible de inmediato para los usuarios finales, muy distinto a como era años atrás.

\subsection{Everything as a service (XaaS)}

El concepto XaaS es una mezcla de todos los modelos anteriormente descritos, donde a su vez se mezclan los diferentes tipos de nubes participativas, formando así una nube híbrida. 


\section{Elección de un modelo}

Ante la variedad de modelos, ¿qué modelo de servicio en la nube es adecuado? Las pymes de hoy en día centran sus operaciones en sus productos y los procesos involucrados, no ven a su empresa desde una arquitectura de servicios. Cuando esta visión cambie, las mismas empresas podrán brindar nuevos servicios a sus clientes basados en despliegues de servicios (laaS, PaaS y SaaS) mucho más sencillos y rápidos de implementar. Con el paso del tiempo, ellas podrán desplegar aplicaciones y servicios que utilizan todos los modelos de servicio.

¿Cómo saber si determinado servicio aplica para estos modelos? Por ejemplo, ¿se debería elaborar un programa propio, comprar una aplicación de escritorio o pagar por una solución SaaS que proporcione la funcionalidad bajo demanda? Si el servicio no es una competencia central, SaaS suele ser una muy buena alternativa a la elaboración, ya que el servicio es asequible, maduro y cumple con los requerimientos del negocio. Las pymes suelen olvidarse de que no deben tercerizar su competencia central, esa que le permite sostener su ventaja competitiva, pues estaría exponiendo el éxito de la empresa. Esa es una regla básica en el juego de negocios. Con respecto al PaaS y al laaS, la decisión está determinada por los requisitos de rendimiento y escalabilidad de la aplicación. Las soluciones PaaS tienen limitaciones en su capacidad para lograr escala muy alta debido a que estas plataformas deben proporcionar autoescala y capacidades de tolerancia a fallos para todos los inquilinos de la plataforma.

\section{Soluciones disponibles en cloud computing}

Actualmente muchas empresas están usando cloud computing, desde las grandes corporaciones hasta las denominadas SMB (small and medium business), mejor conocidas como pymes en nuestro entorno. La variedad de aplicaciones y usos de diferentes recursos y soluciones en la nube en las empresas brindan una idea de cómo aprovechar el uso de cloud computing en los diferentes tipos de negocio. Según el International Data Corporation (IDC), las soluciones en nubes públicas y privadas son cada vez más populares entre las empresas de tamaño mediano. En el año 2013, aproximadamente 3 de cada 10 empresas medianas en todo el mundo planearon implementar soluciones en la nube pública, y 2 de cada 10 empresas de tamaño mediano planearon añadir soluciones de nube privada. Asimismo, se espera que en las pequeñas y medianas empresas (SMB) el gasto en soluciones en la nube pueda crecer en casi un $20 \%$ anual en los próximos cinco años. ¿Pero qué tipo de soluciones se pueden desarrollar con la cloud computing? En la figura 2 se presenta un listado de soluciones disponibles en este modelo. 
Figura 2. Mapeo de soluciones clave en cloud computing

\section{Primary Market IDC IT Product Taxonomy}

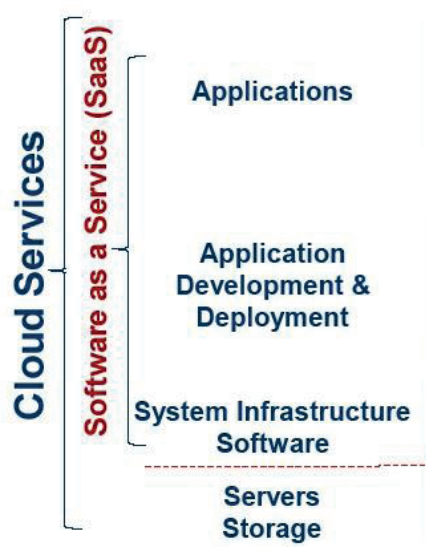

\section{NIST "Taxonomy"}

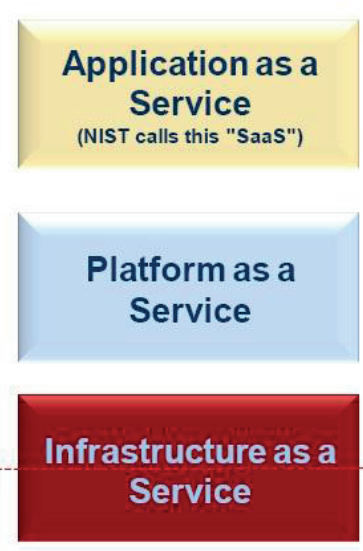

\section{Secondary Market IDC IT Product Taxonomy}

- Collaborative Applications

- Content Applications

- Enterprise Resource Management Applications

- Supply Chain Management Applications

- Operations and Manufacturing Applications

- Engineering Applications

Customer Relationship Management Applications

- Application Development Software

- Application Server Middleware

- Data Access, Analysis, and Delivery

- Information \& Data Management

- Integration \& Process Automation

Middleware

- Other Application Dev and Deployment

- Quality \& Life-Cycle Tools

- Enterprise Portals

- System and Network Management

- Security Applications

- Systems Software

- Servers

- Storage

- Networks

Fuente: IDC, 2013, p. 2.

Asimismo, Cisco Systems (2013) predice que el mercado comercial de servicios para las pymes en Estados Unidos, atendido por los operadores, crecerá a más de 200 billones de dólares al 2015. Es aconsejable aclarar que las SMB en Estados Unidos son empresas que tienen hasta 999 empleados, distintas a nuestra realidad, que son de hasta 50 empleados. Sin embargo, se ve claramente cómo pueden aprovechar sus beneficios a toda escala. En nuestro medio este mercado mejorará con el ingreso de nuevos competidores en el rubro de telecomunicaciones, lo cual traerá mejores ofertas al segmento empresarial. Para explicar el comportamiento de las SMB en Estados Unidos frente a la tecnología de la información, en la tabla 2 se presenta el resumen.

En la tabla 3 se muestra un listado de los principales vendedores de soluciones de cloud computing. 
Tabla 2. Naturaleza de comportamiento de los tipos de empresa frente a las TI

\begin{tabular}{|c|c|c|c|}
\hline $\begin{array}{l}\text { Tipo de } \\
\text { empresa }\end{array}$ & Muy pequeña (1-19) & Pequeña (20-29) & Mediana (100-999) \\
\hline Ubicación & - Básicamente una ubicación & $\begin{array}{l}\text { - Una ubicación y pocas } \\
\text { filiales }\end{array}$ & $\begin{array}{l}\text { - Disperso } \\
\text { geográficamente }\end{array}$ \\
\hline Recursos TI & $\begin{array}{l}\text { - Limitado o sin recursos TI } \\
\text { - Mínima influencia de } \\
\text { profesionales en TI }\end{array}$ & $\begin{array}{l}\text { - Muy pocos recursos TI } \\
\text { - } 17 \% \text { se han dedicado a } \\
\text { tiempo completo a TI } \\
\text { - Usan un proveedor local } \\
\text { de TI }\end{array}$ & $\begin{array}{l}\text { - Pequeño pero } \\
\text { dedicado a TI } \\
\text { • } 75 \% \text { se han dedicado a } \\
\text { tiempo completo a TI. }\end{array}$ \\
\hline $\begin{array}{l}\text { Compra de } \\
\text { servicios TI }\end{array}$ & $\begin{array}{l}\text { - A través de web o call } \\
\text { center por el dueño, con } \\
\text { requerimientos limitados }\end{array}$ & $\begin{array}{l}\text { - A través de web o call } \\
\text { center por el dueño o ge- } \\
\text { rente, con requerimiento } \\
\text { de sus colaboradores }\end{array}$ & $\begin{array}{l}\text { - Personal TI contratado } \\
\text { a tiempo parcial }\end{array}$ \\
\hline $\begin{array}{l}\text { Motivo de } \\
\text { compra TI }\end{array}$ & $\begin{array}{l}\text { - El negocio lo requiere } \\
\text { - Precio y recordación de } \\
\text { la disponibilidad de los } \\
\text { proveedores de servicio }\end{array}$ & $\begin{array}{l}\text { - Eficiencia operacional } \\
\text { - Seguridad }\end{array}$ & $\begin{array}{l}\text { - Continuidad del } \\
\text { negocio y apuntar } \\
\text { hacia el crecimiento de } \\
\text { los ingresos } \\
\text { - Seguridad y } \\
\text { escalabilidad }\end{array}$ \\
\hline & $\longrightarrow$ & & $\longrightarrow$ \\
\hline $\begin{array}{l}\text { Compor- } \\
\text { tamiento } \\
\text { frente a TI }\end{array}$ & $\begin{array}{l}\text { - Aspecto secundario al mo- } \\
\text { mento de iniciar un nuevo } \\
\text { negocio } \\
\text { - Conocimiento limitado de } \\
\text { las opciones en tecnología } \\
\text { y proveedores de servicio } \\
\text { - Productos específicos } \\
\text { - Se resuelven los problemas } \\
\text { con los actuales servicios }\end{array}$ & $\begin{array}{l}\text { - Función de negocios } \\
\text { secundaria } \\
\text { - No es familiar con la } \\
\text { tecnología y servicios } \\
\text { - Soluciones simples y } \\
\text { actuales }\end{array}$ & $\begin{array}{l}\text { - Tl es la principal } \\
\text { función } \\
\text { - Al tanto de las nuevas } \\
\text { tecnologías, pero no } \\
\text { del valor del negocio } \\
\text { - Soluciones no tan } \\
\text { costosas con gran } \\
\text { funcionalidad de } \\
\text { negocio }\end{array}$ \\
\hline
\end{tabular}

Fuente: Adaptado de Cisco, 2013, p. 2.

Tabla 3. Principales vendedores de servicios As a service

\begin{tabular}{|c|c|}
\hline Principales vendedores & Otros proveedores de servicio \\
\hline Amazon Web Services Inc. & América Móvil \\
\hline Google Inc. & Cisco Systems Inc. \\
\hline IBM Corp. & HP Co. \\
\hline Microsoft Corp. & Oi \\
\hline Salesforce.com Inc. & Oracle Corp. \\
\hline Rackspace Hosting Inc. & SAP AG \\
\hline Verizon Communications Inc. & Telefónica S. A. \\
\hline
\end{tabular}

Fuente: Adaptado de Researchbeam.com (2014). 


\section{4. ¿Por qué elegir cloud computing?}

Bien reza el dicho: "si no puedes medir, no puedes gestionar y si no puedes gestionar no puedes gerenciar". Por lo tanto, es necesario conocer las diferentes perspectivas para decidir sobre este tipo de soluciones.

\subsection{La perspectiva de Administración General: El ROI debe prevalecer}

El retorno de la inversión (ROI), clásico enfoque típicamente asociado con el ahorro de costos, es la medida tradicional para cualquier decisión de inversión. Si el retorno compensa el costo del capital, entonces vale la pena hacer la inversión. El primer paso se ve en el ahorro de costos de licencias y actualizaciones de hardware, que implicaría una opción local. Idealmente, hay suficientes beneficios financieros para justificar una solución cloud. Una vez que se toma una decisión, los resultados deben ser rastreados en el tiempo para confirmar que obtienen los beneficios deseados.

\subsection{La perspectiva de las tecnologías de información: mejorar aspectos de flexibilidad y agilidad organizacional}

Los aspectos financieros de la computación en nube y SaaS son, ciertamente, convincentes: pagar solo por lo que necesitan, sin tener que poseer capacidades que no pueden ser utilizadas completamente. Beneficios como la facilidad de manejo, las versiones compatibles de software utilizado en toda la organización y la velocidad con la que se pueden agregar nuevos usuarios y mejoras implementadas. Estos beneficios serán de interés para los administradores de IT, pero también pueden hacer que la empresa sea mucho más ágil, asegurando que los usuarios confíen en los mismos recursos. El ahorro de estos beneficios se reflejará en la reducción de costos, por ejemplo las horas extraordinarias; sin embargo, las mejoras en la eficiencia pueden ser difíciles de cuantificar. Otras medidas — como el tiempo de implementar nuevos procedimientos o la capacidad de coordinar las diferentes actividades de manera más eficiente — también podrían ser tomadas en cuenta.

\section{Consideraciones adicionales sobre la cloud computing}

Cabe reflexionar si una solución en la nube podrá facilitar el logro de objetivos de la empresa. Antes de examinar las fortalezas y debilidades de las ofertas de servicios en la nube, las medianas empresas necesitan establecer algunas pautas básicas que faciliten el proceso de evaluación. El IDC (2013) ha encontrado que casi el 60 \% de las medianas empresas en el mundo están de acuerdo $(6$ o 7 en una escala de 7 
puntos de acuerdo/desacuerdo) y consideran a la tecnología avanzada como una herramienta competitiva que es utilizada como un recurso estratégico.

Por tanto la cloud computing puede contribuir a la función de la tecnología como un diferenciador competitivo, con la suficiente tecnología innovadora y disponible de una manera escalable a través de su organización. En especial para empresas que pueden beneficiarse de soluciones listas para implementar de manera oportuna, teniendo los recursos disponibles en la nube, los cuales permitirán mejorar el rendimiento en un área de aplicación específica. El beneficio inmediato de las soluciones en la nube será de carácter táctico, proporcionando las economías de costos y despliegue; más en el largo plazo, las implicancias estratégicas de las capacidades de la nube serán aún más valiosas, ya que responder rápidamente a los requerimientos de los clientes a través de una tecnología altamente escalable permitirá mejorar la posición competitiva empresarial.

También será importante considerar el número de sedes de una empresa, sumado a su actual infraestructura de tecnología de información, en cualquier implementación de soluciones en la nube, ya que las empresas con un único local emplean menos tiempo para coordinar sobre la gestión de los recursos de tecnología que aquellas con múltiples sedes. Además, son pocas las empresas que tienen personal a tiempo completo para el soporte de las tecnologías de información; esta situación puede complicar el mantenimiento general, la instalación de nuevo software y las actualizaciones respectivas; con el riesgo potencial de dar soporte a varias versiones de la misma suite de programas en cualquier momento. Una ventaja importante de las aplicaciones SaaS es que todos los usuarios ejecutan la última versión de aplicaciones hospedadas, simplificando el soporte de múltiples ubicaciones. En esencia, mientras más localizaciones cuente su empresa, más diverso suele ser su entorno de tecnologías de información; en este aspecto la nube es muy útil en la coordinación y gestión para el despliegue de aplicaciones en toda la empresa. El número de sedes y su proximidad física pueden influir en la decisión de apostar por una nube pública frente a una privada. Para las grandes empresas con múltiples sedes, puede ser atractivo utilizar una nube privada para la transferencia segura de datos bajo el control exclusivo de la empresa. Una nube privada también se puede extender a los socios u otros terceros para proporcionar una ventaja competitiva, aun cuando una nube pública puede representar una alternativa de menor costo.

Sin embargo, la integración de los recursos de tecnologías de información no se encuentra entre las principales preocupaciones de las empresas, sino hasta que pueda surgir un impacto desagradable en la organización. Para las empresas que experimentan grandes cambios, las contrataciones de servicio en la nube pueden iniciar una etapa de mayor flexibilidad organizacional para el mañana. Por ejemplo, las soluciones en la nube pueden permitir a las empresas medianas agregar o 
probar nuevas aplicaciones o procesos sin tener que ampliar su infraestructura tecnológica. Esto, en síntesis, puede ayudar a impulsar la innovación y permitir a las pymes ser más ágiles en respuesta a las condiciones cambiantes.

Otro beneficio es la mejora de la productividad de los trabajadores; esta es una razón clave para aumentar la inversión en tecnología, pues esta puede permitir el acceso a funcionalidades avanzadas de redes mediante soluciones basadas en la nube. Desde la perspectiva del retorno de la inversión (ROI), para el trabajo realizado por un trabajador móvil puede ser convincente; en ese caso, solo contaría como mejora las acciones que tengan relación con sus clientes de manera directa y tangible, como apoyarlos en el cierre de negocios, acceso en tiempo real a las posiciones de inventario o inteligencia competitiva. Conforme pase el tiempo, el trabajo remoto se volverá más común en las empresas de tamaño mediano, por tanto el argumento de los recursos basados en la nube será más convincente.

Pero ¿qué fuerzas externas están alentando la adopción de computación en nube? Mientras que cualquier inversión en tecnología debe basarse en las necesidades internas, los recursos disponibles, los recursos externos también deben ser reconocidos y examinados. El entorno competitivo y el entorno regulatorio pueden ser cambiantes y ofrecer fuertes incentivos o disuasivos para la adopción de diferentes soluciones de computación en nube; asimismo, el avance de la tecnología móvil y la consolidación de toda la información en un solo dispositivo. Para algunos, el acceso oportuno a la información relevante de su empresa anulará cualquier preocupación; sin embargo, para otros, la seguridad de las transacciones y una buena administración serán primordiales; estas preocupaciones seguirán incrementándose conforme la cloud computing se expanda, pero se extinguirán, así como sucedió con la preocupación que alguna vez desalentó el uso de tarjetas de crédito para las compras en línea.

\section{Conclusiones y recomendaciones}

La cloud computing puede ser una convincente y atractiva forma de adquirir capacidades y reducir los costos; sin embargo, la pequeña y mediana empresa necesita acercarse a las implementaciones en la nube con los ojos abiertos y ser sensibles a algunas cuestiones clave para asegurar que los beneficios esperados se concreten, las que se presentan a continuación:

a) Integración de las prioridades del negocio y las prioridades de las tecnologías de información con la estrategia de despliegue de la nube, es decir, los objetivos estratégicos de la empresa o de la unidad de negocio necesitan estar claros y entendidos, y a su vez ser soportados por la tecnología. 
b) Maximización en la flexibilidad y agilidad de los negocios relacionados con la computación en nube para un impacto máximo. El beneficio real de la cloud computing es su papel potencialmente transformador, proporcionando acceso a los recursos consistentes en toda la organización, con certeza y en forma oportuna.

c) Disponibilidad 24x7, y crecimiento de la solución desplegada gracias a la alta escalabilidad en el modelo.

d) La integración de los recursos de la nube y lo existente fuera de ella es a largo plazo; con ello se busca que las empresas puedan agilizar sus procesos vitales y de negocio a través del soporte tecnológico, lo cual a la vez le dará una ventaja competitiva. Con el tiempo, sin embargo, las pequeñas empresas deberían anticipar y alentar este desarrollo para el aprovechamiento de los recursos locales y la maximización de beneficios dirigidos al crecimiento de su negocio.

\section{Referencias}

Carlo, A., Biancolino, C., \& Maccari, E. (2013). Cloud computing and information technology strategy. Journal of Technology Management \& Innovation, 8, Special issue, 178-188.

Cisco System (2013). What do SMBs want from commercial-services providers? Insights from Cisco's U.S. research on SMB Services delivery. Recuperado de https://www.cisco.com/web/about/ac79/docs/sp/SMB-Cloud-Survey.pdf

Instituto Nacional de Estadística e Informática - INEI (2014). Resultados de la encuesta de micro y pequeña empresa, 2013. Lima, Perú: INEl.

International Data Corporation - IDC (2013a). Cloud computing in the midmarket: Assessing the options in 2013. Recuperado de http://idcdocserv.com/995

International Data Group (2013b). Gartner advierte sobre la confusión reinante en el uso del concepto de cloud computing. Recuperado de www.idg.es/ macworld/content.asp?idn=71975

Researchbeam (2013). Recuperado de http://www.researchbeam.com/cloudcomputing-in-latin-america-2014-2018-market

Yrigoyen, M., \& Torres, C. (2011). Servicios educativos mediante la utilización de tecnologías de cloud computing. Interfases, 4, 11-38. Recuperado de http:// fresno.ulima.edu.pe/sf/rd_bd6500_001.nsf/EdAnterior/D878BCAF122B3DA 6052579B400674F13/\$file/1.PDF 


\section{Anexo}

Tabla 4. Constitución de las pymes en el Perú, 2013

\begin{tabular}{|l|c|}
\hline $\begin{array}{l}\text { Tipo de organización } \\
\text { jurídica }\end{array}$ & $\%$ \\
\hline Persona natural & 45.8 \\
\hline $\begin{array}{l}\text { Sociedad Anónima } \\
\text { Cerrada }\end{array}$ & 26.4 \\
\hline $\begin{array}{l}\text { Empresa Individual } \\
\text { de Responsabilidad } \\
\text { Limitada }\end{array}$ & 15.0 \\
\hline $\begin{array}{l}\text { Sociedad Comercial } \\
\text { de Responsabilidad } \\
\text { Limitada }\end{array}$ & 10.1 \\
\hline Sociedad Anónima & 2.7 \\
\hline Total & 100.0 \\
\hline
\end{tabular}

\begin{tabular}{|c|c|}
\hline $\begin{array}{c}\text { Condición de } \\
\text { locales }\end{array}$ & $\%$ \\
\hline Alquilado & 50.0 \\
\hline Propio & 40.0 \\
\hline Prestado & 10.0 \\
\hline Total & 100.0 \\
\hline
\end{tabular}

\begin{tabular}{|c|c|}
\hline $\begin{array}{c}\text { Nivel de educación } \\
\text { alcanzado }\end{array}$ & $\%$ \\
\hline $\begin{array}{c}\text { Secundaria com- } \\
\text { pleta }\end{array}$ & 33.4 \\
\hline $\begin{array}{c}\text { Superior universita- } \\
\text { ria completa }\end{array}$ & 23.0 \\
\hline $\begin{array}{c}\text { Superior no univer- } \\
\text { sitaria completa }\end{array}$ & 16.2 \\
\hline $\begin{array}{c}\text { Superior universita- } \\
\text { ria incompleta }\end{array}$ & 8.4 \\
\hline $\begin{array}{c}\text { Superior no univer- } \\
\text { sitaria incompleta }\end{array}$ & 6.9 \\
\hline Secundaria incom- \\
pleta
\end{tabular}

Micro y pequeña empresa que pertenecían a alguna organización o agrupación con fines empresariales y motivos para asociarse, 2012 (Distribución porcentual)

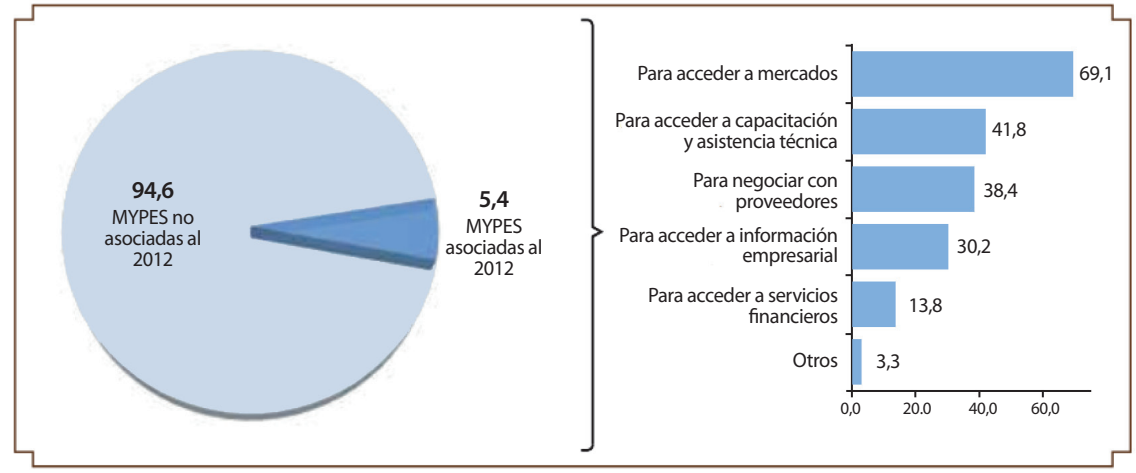


Figura 3. Cambios en las proyecciones de crecimiento del PBI 2014

Fuente: INEI, 2014, p. 14.

Tabla 5. Micro y pequeña empresa que accedieron a financiamiento, según ciudad, 2012

\begin{tabular}{|l|c|c|c|}
\hline \multirow{2}{*}{ Ciudad } & \multirow{2}{*}{$\begin{array}{c}\text { Total } \\
\text { Mype }\end{array}$} & \multicolumn{2}{c|}{$\begin{array}{c}\text { Mypes que accedieron al } \\
\text { financiamiento }\end{array}$} \\
\cline { 3 - 4 } & & Absoluto & $\%$ \\
\hline Total & 11937 & 5145 & 43.1 \\
\hline Lima - Callao & 9373 & 3821 & 40.8 \\
\hline Arequipa & 839 & 370 & 44.1 \\
\hline Ayacucho & 48 & 30 & 62.5 \\
\hline Chiclayo & 252 & 139 & 55.3 \\
\hline Cusco & 163 & 117 & 72.0 \\
\hline Huancayo & 195 & 129 & 66.4 \\
\hline Iquitos & 97 & 39 & 39.7 \\
\hline Juliaca & 147 & 94 & 63.9 \\
\hline Piura & 114 & 68 & 59.4 \\
\hline Trujillo & 709 & 338 & 47.7 \\
\hline
\end{tabular}

Fuente: INEI, 2014, p. 55. 